Supporting Information

\title{
Foldable Thermoelectric Materials: Improvement of the Thermoelectric Performance of Directly Spun CNT Webs by Individual Control of Electrical and Thermal Conductivity
}

Cheng Jin $\mathrm{An}^{1}$, Young Hun Kang ${ }^{1}$, A-Young Lee ${ }^{2}$, Kwang-Suk Jang ${ }^{1}$, Youngjin Jeong ${ }^{2}$, and Song Yun Cho ${ }^{1 *}$

${ }^{1}$ Division of Advanced Materials, Korea Research Institute of Chemical Technology, 141 Gajeong-ro, Yuseong-gu, Daejeon 34114, Republic of Korea

${ }^{2}$ Department of Organic Materials and Fiber Engineering, Soongsil University, 369 Sangdo-ro, Dongjak-gu, Seoul 06978, South Korea

KEYWORDS: CNT web; gold dopant; conducting polymers; thermoelectrics; flexible thermoelectric generator

"E-mail: $\underline{\text { scho@ } \text { krict.re.kr }}$ 

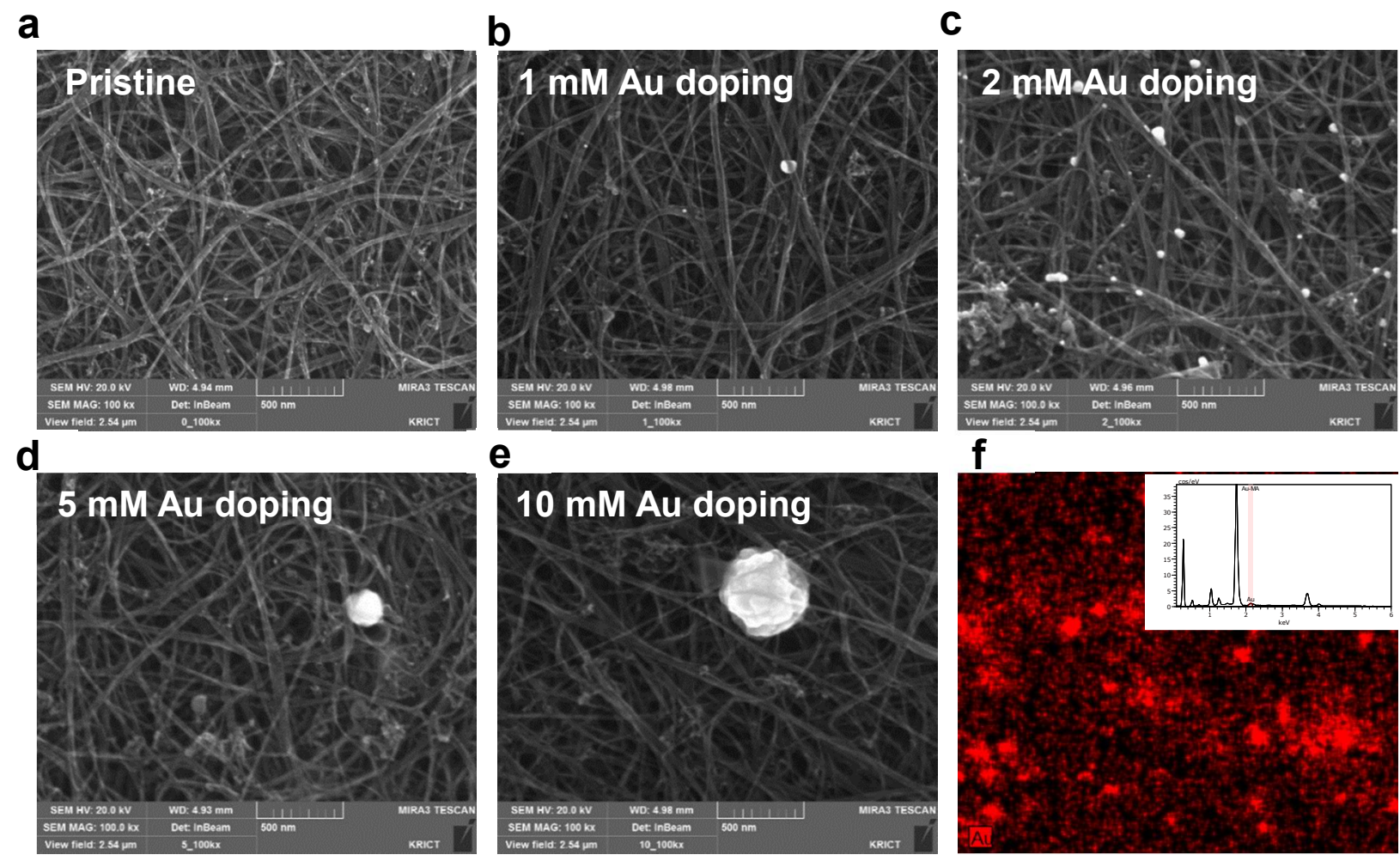

Figure S1. SEM images of Au-doped CNT webs with different Au dopant concentrations. (a) Pristine CNT web, (b) $1 \mathrm{mM}$ Au doped CNT web, (c) 2 mM Au doped CNT web, (d) 5 mM Au doped CNT web, (e) $10 \mathrm{mM}$ Au doped CNT web, and (f) EDXS mapping image with the EDXS spectrum in the inset of $2 \mathrm{mM}$ Au doped CNT web. The Au coating was controlled by adjusting the concentration of the Au-dopant solution while maintaining a constant dipping time. The size of the Au NPs increased as the Au dopant concentration increased. 
a

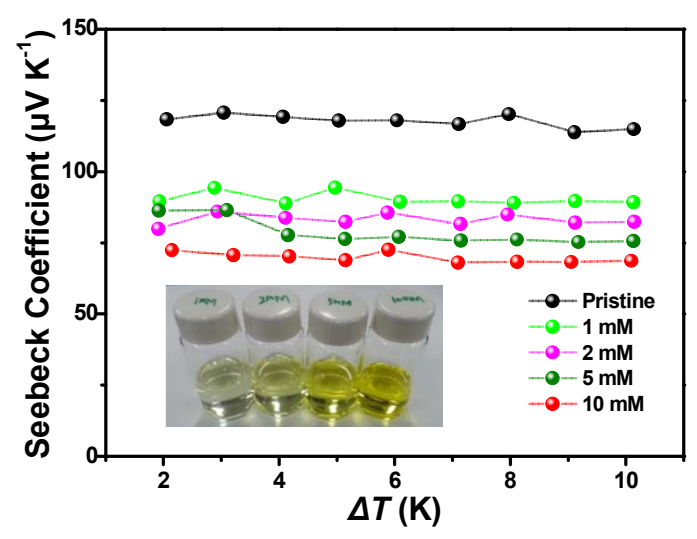

b

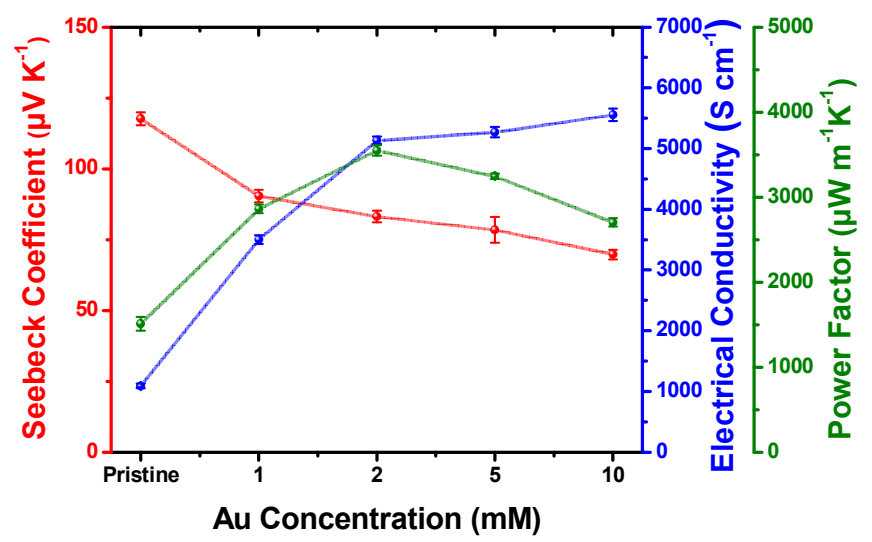

Figure S2. (a) Seebeck coefficient versus temperature for Au-doped CNT webs depending on Au dopant concentrations. The inset is a photograph of the Au dopant solutions at different concentrations as designated. (b) Seebeck coefficient (red filled circles), electrical conductivity (blue filled circles), and corresponding power factor (green filled circles) versus Au dopant concentration. The Seebeck coefficient is inversely proportional to the electrical conductivity. 

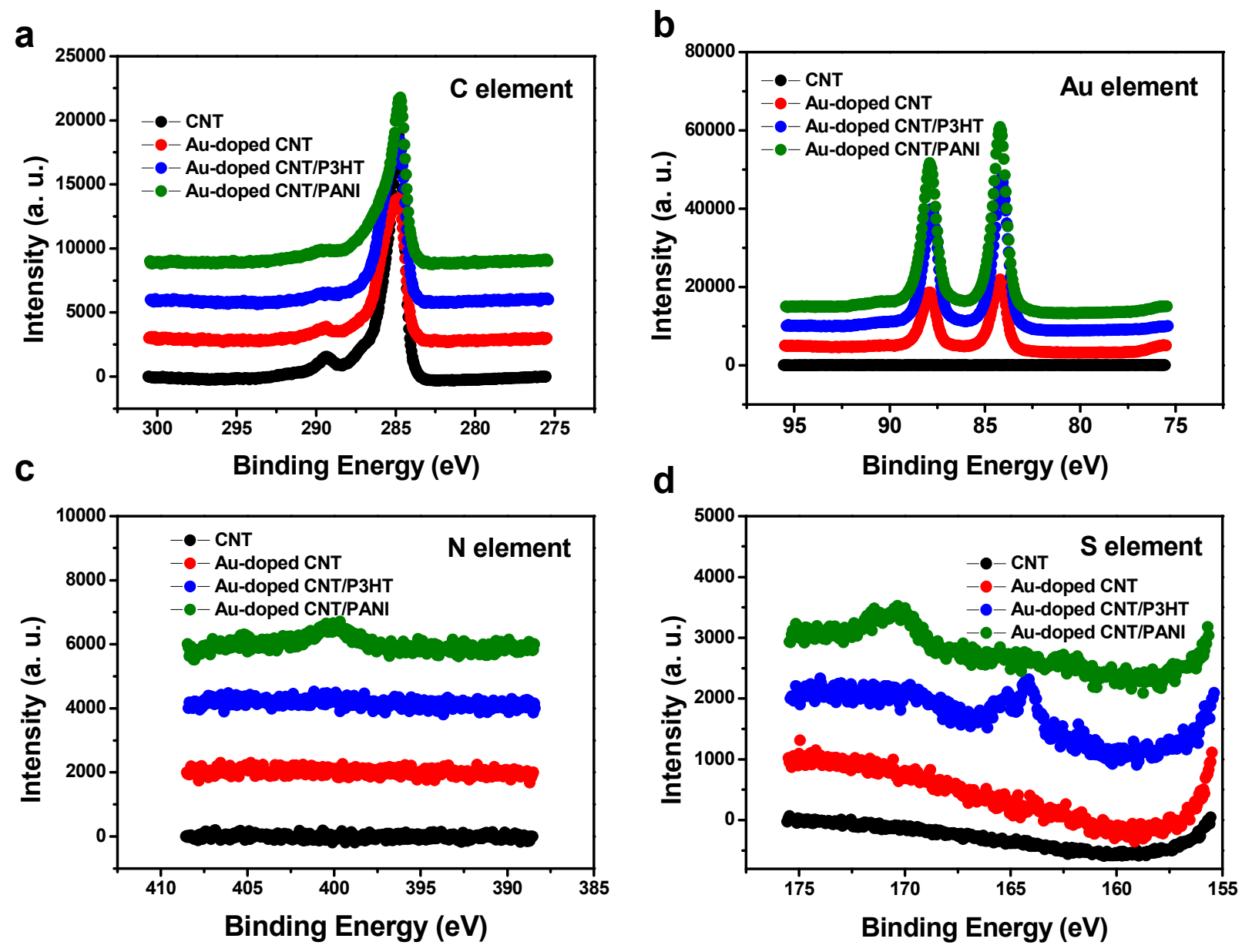

Figure S3. XPS spectra for CNT web, Au-doped CNT web, Au-doped CNT/P3HT web, and Au-doped CNT/PANI web. The peaks at 284.8, 84.2, 400.9, and 164.1 correspond to the $\mathrm{C}$ $1 s$, Au 1s, N 1s, S1s in the webs. (a) All samples show intensive carbon peaks and shadow peak was decreased with Au treatment and polymer infiltration. (b) Au was detected from all samples except for pristine CNT web. (c) Nitrogen was detected from Au-doped CNT/PANI webs, confirming the presence of PANI. (d) Sulfur was detected from Au-doped CNT/P3HT web, confirming P3HT in the web. 
a

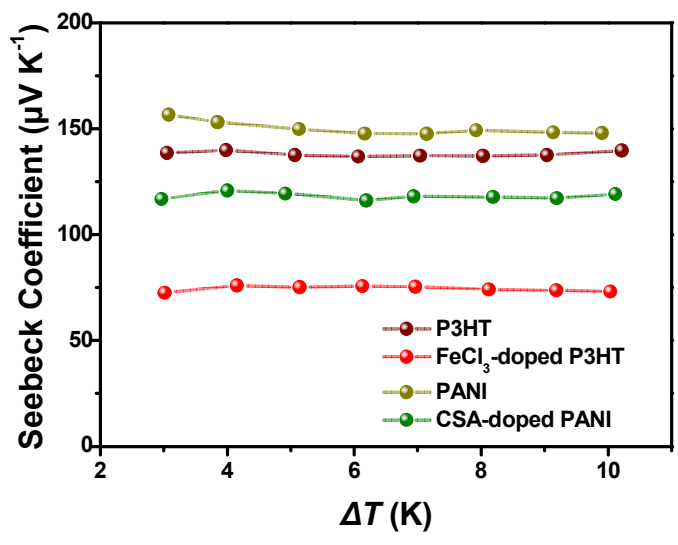

b

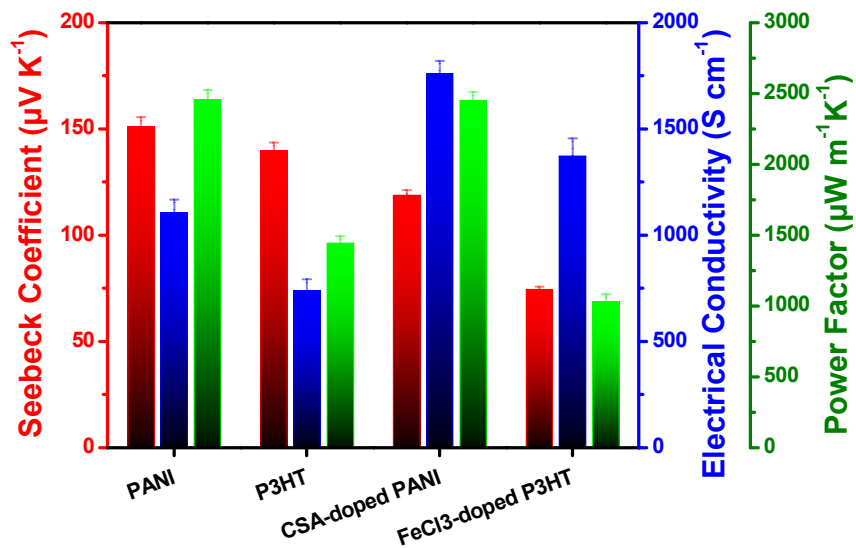

Figure S4. (a) Seebeck coefficient versus temperature depending on the various treated polymers on Au-doped CNT webs. (b) Seebeck coefficient (red column), electrical conductivity (blue column), and corresponding power factor (green column) versus the treated conducting polymers. The PANI treated Au-doped CNT web exhibits the highest power factor among other polymers. 


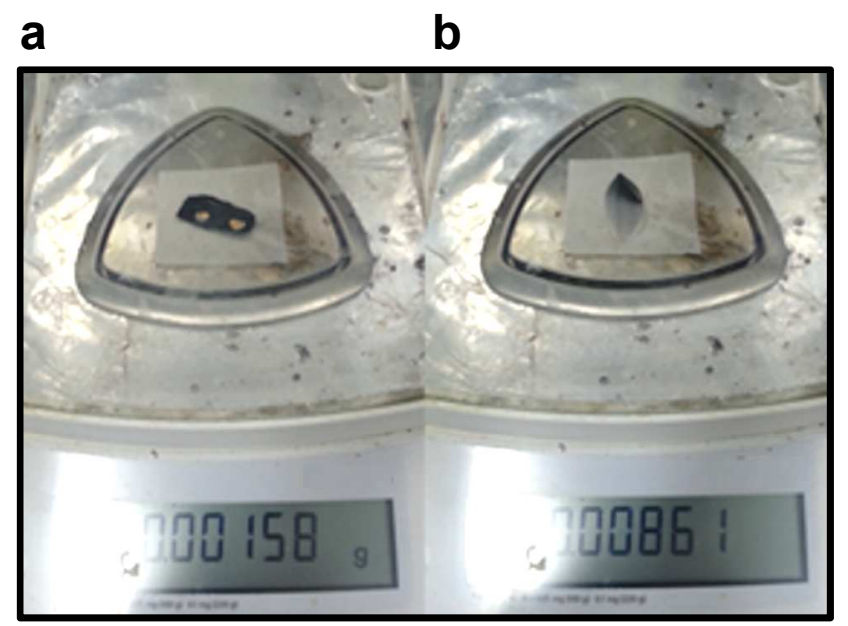

Figure S5. Weight measurement of (a) Au-doped CNT/PANI web and (b) feather for the comparison. Au-doped CNT/PANI web exhibits 5 times lighter weight than feather with similar size. 

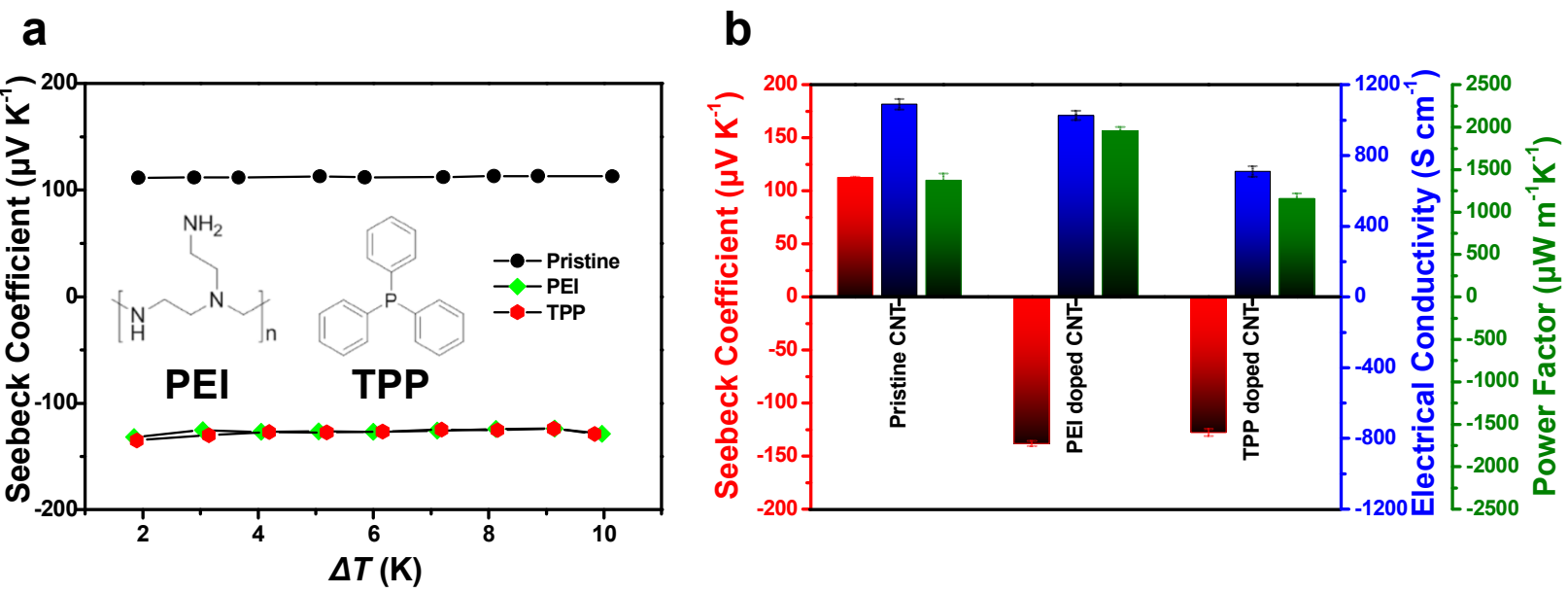

Figure S6. (a) Seebeck coefficient versus temperature for n-type CNT webs doped with polyethyleneimine (PEI) and triphenylphosphine (TPP). Inset presents the molecular structure of n-type dopants. (b) Seebeck coefficient (red column), electrical conductivity (blue column), and corresponding power factor (green column) versus the different n-type dopants. PEI-doped CNT web shows higher power factor than TPP with higher electrical conductivity. 
a

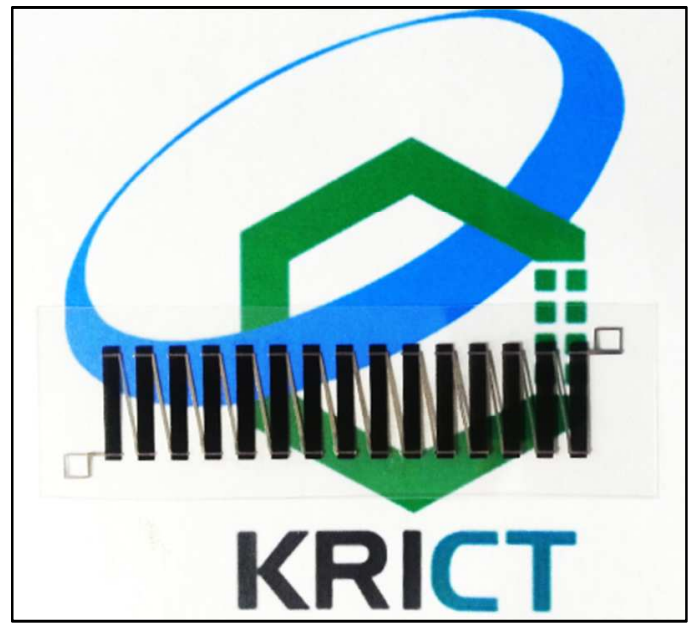

b

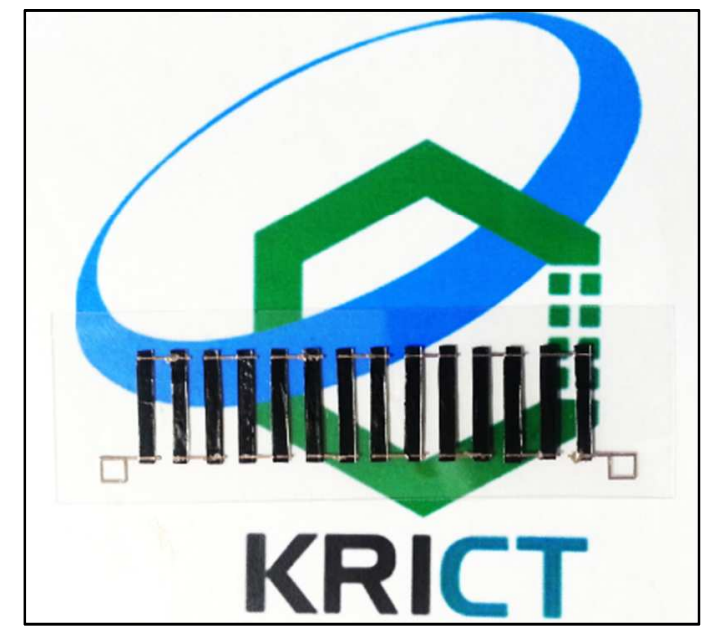

Figure S7. Photographs of (a) flexible p-type TEG, which is composed of 14 legs of Audoped CNT/PANI webs on a PET substrate and (b) flexible $p$ - $n$ TEG where the $p$-type legs are alternatively connected with PEI-doped $\boldsymbol{n}$-type legs in serials. 


\section{a}

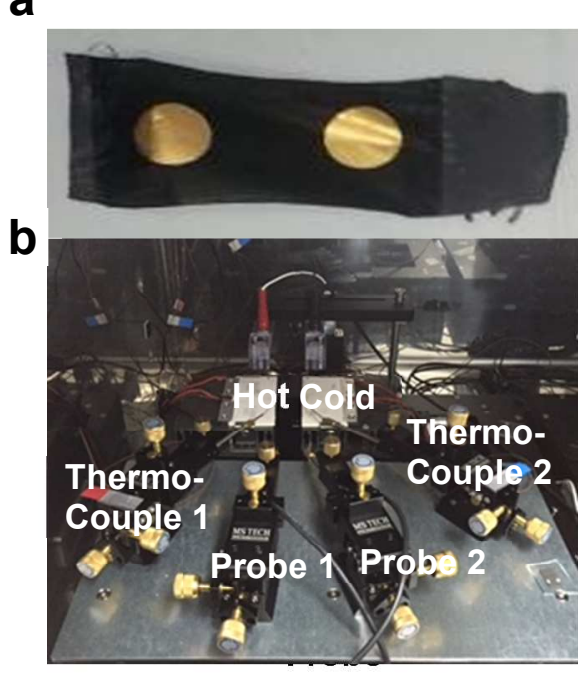

C
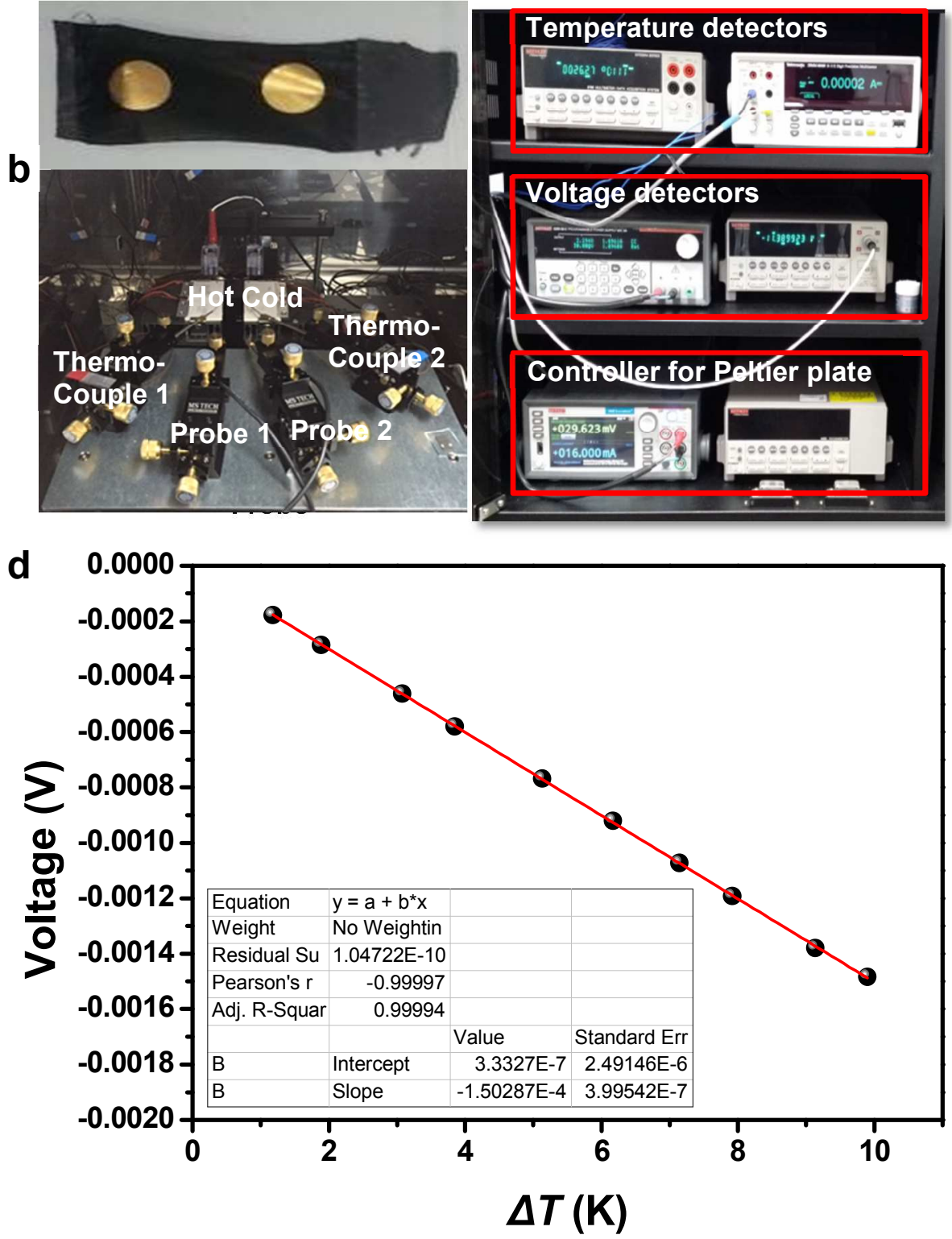

Figure S8. (a) Optical image of sample prepared for Seebeck coefficient measurement. (b) Experimental setup for the Seebeck coefficient measurement. (c) Detectors for temperature and voltage and temperature controller for Peltier plate. (d) A typical curve of thermoelectric voltage versus temperature gradient for Au-doped CNT/PANI web. 
Table S1. Thermal Conductivity, $\kappa$, and Related Data of CNT Webs at $300 \mathrm{~K}$

\begin{tabular}{|c|c|c|c|c|}
\hline CNT web & $\begin{array}{c}\alpha \\
{\left[\mathrm{mm}^{2} \mathrm{~s}^{-1}\right]}\end{array}$ & $\begin{array}{c}C_{\mathrm{p}} \\
{\left[\mathrm{J} \mathrm{g}^{-1} \mathbf{K}^{-1}\right]}\end{array}$ & $\begin{array}{c}\rho \\
{\left[\mathrm{g} \mathrm{cm}^{-3}\right]}\end{array}$ & $\begin{array}{c}k \\
{\left[\mathbf{W} \mathbf{m}^{-1} \mathbf{K}^{-1}\right]}\end{array}$ \\
\hline Pristine CNT web & $18 \pm 0.2$ & $0.77 \pm 0.03$ & $0.41 \pm 0.03$ & $5.68 \pm 0.25$ \\
\hline Au-doped CNT web & $20.9 \pm 0.3$ & $0.79 \pm 0.04$ & $0.43 \pm 0.03$ & $7.09 \pm 0.32$ \\
\hline $\begin{array}{c}\text { Au-doped } \\
\text { CNT/P3HT web }\end{array}$ & $8.98 \pm 0.3$ & $1.01 \pm 0.03$ & $0.45 \pm 0.04$ & $4.53 \pm 0.21$ \\
\hline $\begin{array}{c}\text { Au-doped } \\
\text { CNT/PANI web }\end{array}$ & $8.06 \pm 0.2$ & $1.02 \pm 0.02$ & $0.44 \pm 0.03$ & $3.61 \pm 0.15$ \\
\hline
\end{tabular}

